\title{
Gegen den Zufall anschreiben. Zu Tradition und Funktion des unzuverlässigen Erzählens in Alfred Anderschs Efraim (1967)
}

\author{
Matthias Aumüller
}

Zusammenfassung Der Beitrag besteht aus drei Teilen. Zuerst wird erörtert, dass und inwiefern Anderschs Roman Efraim unzuverlässig erzählt ist. Die Zufallstheorie, die er über weite Strecken vertritt, soll als falsche Theorie verstanden werden. Im zweiten Teil wird dargelegt, welcher Tradition Andersch mit seiner Konzeption narrativer Unzuverlässigkeit folgt. Es ist nicht die Tradition Samuel Becketts, auf den im Roman wiederholt angespielt wird, sondern diejenige Max Frischs, auf den sich Andersch in Interview-Äußerungen auch selbst beruft. Im letzten Abschnitt werden drei Funktionen vorgestellt, die mit der Unzuverlässigkeit des Erzählers Efraim verknüpft sind, eine kognitive, weil sein reflektierendes Schreiben ihm einen Erkenntnisfortschritt beschert, eine emotionale, weil er auf diese Weise mit seiner Verzweiflung umgehen kann, und eine transtextuelle Funktion, die darin besteht, den Widerspruch der Leser zu provozieren.

Efraim ist ein Roman mit einer seltsamen Rezeptionsgeschichte. Aufgrund der hohen Bekanntheit seines Autors schien er sich anfangs ebenso gut zu verkaufen wie die früheren Romane Sansibar oder der letzte Grund (1957) und Die Rote (1960). Schon nach kurzer Zeit ließ der Verlag eine zweite Auflage drucken (vgl. Reinhardt 1990, S. 441). Die Kritik war gespalten. Die einen lobten den Roman, andere verrissen ihn. Aber es wurde über ihn geredet, und Andersch erhielt auf Empfehlung von Nelly Sachs den Dortmunder Nelly-Sachs-Preis. Doch dann brach der Absatz ein, und im Sommer erfuhr Andersch, dass fast die ganze zweite Auflage remittiert worden war (vgl. Reinhardt 1990, S. 451).

M. Aumüller $(\bowtie)$

Departement für Germanistik,

Universität Fribourg,

Freiburg, Schweiz

E-Mail: matthias.aumueller@unifr.ch 
Damit war das Schicksal des Romans besiegelt. Fortan führte er ein germanistisches Fußnotendasein - bis sich W. G. Sebald (1999 [1993]) seiner annahm und den Roman wie auch seinen Autor auf das heftigste kritisierte. In Verbindung mit den bis zur Publikation von Stephan Reinhardts Andersch-Biographie unsichtbar gebliebenen Flecken in Anderschs Leben erlangte der Roman zumindest im Rahmen der literarturwissenschaftlichen Holocaust-Forschung eine gewisse Bedeutung, die sich in einer umfangreichen Rezeption mit durchaus wechselhaften Einschätzungen niederschlug. Die Debatte um den Roman scheint inzwischen weitgehend abgeschlossen zu sein. Eine Bilanz wurde indes noch nicht gezogen. In literaturwissenschaftlicher Hinsicht lässt sich immerhin eine Lehre daraus ziehen: Hätten die Beteiligten über den Begriff des unzuverlässigen Erzählens verfügt und ihn bei ihren Interpretationen in Erwägung gezogen, hätte die Debatte sicherlich einen anderen Verlauf genommen.

Während ich die Rezeption ausführlich an anderer Stelle darstelle, ${ }^{1}$ möchte ich in diesem Beitrag auf den Kontext der narrativen Unzuverlässigkeit eingehen: auf Fragen nach der Tradition, in die sich Andersch damit stellt, und auf Fragen nach den Funktionen, die das Verfahren in dem Roman erfüllt. Der Beitrag besteht daher aus drei Teilen. Zuerst frage ich danach, inwiefern Anderschs Roman Efraim unzuverlässig erzählt ist. In den beiden Teilen danach frage ich jeweils, warum Efraim so erzählt ist. Aus welchem Grund hat Andersch sich für diese Erzählkonzeption, d. h. für einen Erzähler entschieden, der diese Eigenschaft hat? Was hat ihn dazu veranlasst?

Die Frage „Warum?“ erlaubt mehr als nur eine Antwort und ist daher mehrdeutig. Zum einen lässt sich fragen, woran sich ein Autor orientiert hat. Die Antwort darauf kann vielfältig ausfallen, je nachdem, welche Faktoren von Interesse sind: psychologische, soziologische oder andere. Im vorliegenden Fall ist der Suchbereich die Gegenwartsliteratur zur Zeit Anderschs. Die Antwort besteht aus einer empirischen bzw. literaturhistorischen Hypothese mit probabilistischem Charakter: Gemäß der Quelle $q$ hat sich Andersch wahrscheinlich am literarischen Vorbild $x$ orientiert. Das schließt nicht aus, dass es weitere literarische Vorbilder gibt. Vollständigkeit ist hier nicht der Anspruch. Es geht darum, eine bis auf weiteres wahrscheinliche Hypothese als Erklärungsangebot dafür vorzuschlagen, welches literarische Vorbild Andersch dazu bewogen haben könnte, das Erzählverfahren narrativer Unzuverlässigkeit einzusetzen. Wie sich erweisen wird, zieht die Hypothese weitere Antworten nach sich, da Anderschs Wahl in diesem Zusammenhang auch Auskunft über seine poetologischen Zielsetzungen gibt.

Das führt zugleich zu der anderen Variante der Frage, warum Andersch dieses Erzählverfahren gewählt hat: Welchem Zweck oder welchen Zwecken dient die Erzählkonzeption? Auch die Antworten darauf können unterschiedlich ausfallen. Zunächst lässt sich die Frage wiederum empirisch verstehen: Welchen Zwecken sollte diese Erzählkonzeption Andersch zufolge dienen? Oder, anders gesagt: Was

\footnotetext{
${ }^{1} \mathrm{Vgl}$. Anm. 12.
} 
bezweckte Andersch damit? Was wollte er damit erreichen? Die erste Antwort darauf liefert Erkenntnisse über Anderschs Poetik und gehört insofern zur ersten Kategorie, als diese Poetik in einem literaturgeschichtlichen Traditionszusammenhang steht. Die zweite Antwort darauf gehört jedoch zur zweiten Kategorie, wenn sie werk- oder rezeptionsorientiert aufgefasst wird und die Effekte benennt, die hervorzubringen die spezifische Realisierung des Erzählverfahrens narrativer Unzuverlässigkeit im Roman Efraim geeignet ist.

Somit lässt sich die Frage nach den Zwecken auch als Frage nach den Funktionen auffassen. ${ }^{2}$ Statt der tatsächlichen Ziele des Autors kann man die Funktionen - gleich ob der Autor sie bedachte oder nicht - ermitteln. Dabei lassen sich Funktionen bestimmen, die eine Eigenschaft des Werks, die zur erzählten Welt gehört (wie die unzuverlässig erzählte Zufallstheorie), für eine andere Bezugsgröße in diesem Bereich hat (Efraims Psyche z. B.); sowie solche Funktionen, die diese Eigenschaft mit werkexternen Bezugsgrößen in Verbindung bringt.

Ich habe also mehrere Fragen. Die Antworten nehme ich thesenartig vorweg:

1. Die Frage nach der Tradition (2. Abschnitt): Woran hat sich Andersch orientiert mit seiner Unzuverlässigkeitskonzeption? - Die Antwort besteht in diesem Falle aus einer empirischen bzw. historischen Hypothese mit probabilistischem Charakter: Gemäß der Quellenlage hat sich Andersch am Vorbild Max Frisch und dessen besonderer Erzählweise in Stiller und Homo Faber orientiert.

2. Die Frage nach möglichen Funktionen (3. Abschnitt): a) Welche (intratextuelle) Funktion die narrative Unzuverlässigkeit für Efraim selbst hat, lässt sich daran erkennen, wie sie aufgelöst wird: durch Efraims Einsicht nämlich, dass die Zufallstheorie falsch ist. Ihre erste Funktion ist also die Darstellung eines kognitiven Fortschritts. b) Zugleich dient die falsche Theorie, die Efraims Unzuverlässigkeit ausmacht, der (letztlich unvollkommenen) Bewältigung seiner Verzweiflung. Die zweite Funktion hat demnach einen emotionalen Charakter. c) Schließlich hat die falsche Theorie die extratextuelle Funktion, den Widerspruch des Lesers herauszufordern, sowie die, eine mehrdimensionale, brüchige literarische Figur zu kreieren.

\section{Die Diagnose: George Efraims mimetische Unzuverlässigkeit}

Die Zuschreibung von mimetischer Unzuverlässigkeit an einen Erzähler dient als Erklärung von Ungereimtheiten, die dem Erzähler unterlaufen. Diese Ungereimtheiten oder Anomalien können explizite Widersprüche sein, es können aber auch Absonderlichkeiten sein - die sich aber letztlich auch als Widersprüche

\footnotetext{
${ }^{2}$ Zum Funktionsbegriff vgl. Fricke (1981, S. 88-100) und Zymner (2013, S. 75-112).
} 
reformulieren lassen. Damit die Zuschreibung von mimetischer Unzuverlässigkeit zutrifft, muss eine der expliziten oder impliziten Behauptungen des Erzählers über den Zustand der Welt falsch sein. ${ }^{3}$ Implizit ist eine Behauptung genau dann, wenn der Behauptende das Bestehen eines Sachverhalts $S$ zu verstehen gibt, ohne dass die Prädikation, die das Bestehen von S durch die Zuschreibung einer Eigenschaft an einen Gegenstand ausdrückt, Wort für Wort Bestandteil seiner Rede ist bzw., anders ausgedrückt, wenn der Behauptende das Bestehen eines Sachverhalts $\mathrm{S}$ dadurch zu verstehen gibt, dass er das Bestehen von S mittels Andeutungen ausdrückt, die am ehesten so ausgelegt werden, dass er das Bestehen von S behauptet.

Eine der zentralen Überzeugungen Efraims über die Beschaffenheit der Welt ist, dass alles, was passiert, keinem Plan folgt und dass der Mensch keine Einwirkungsmöglichkeit hat:

Ich hingegen glaube weder an das Schicksal noch an die Vernunft. Es gibt nichts als ein großes Durcheinander. Dinge geschehen oder geschehen nicht, Menschen kommen und gehen, tun dies oder jenes, worauf irgend etwas oder nichts geschieht. Immer ist alles möglich oder unmöglich. Es gibt keine Gesetze und keine Freiheit. $\left(\right.$ E, 17) ${ }^{4}$

Die Formel, auf die er diese Ansicht bringt, ist, dass alles Zufall sei - auch der Holocaust. Die entscheidende Frage ist nun, ob auch wir Leser glauben sollen, dass diese Ansicht Efraims in der Welt des Romans eine (auf diese Welt) zutreffende Ansicht ist. Der Sachverhalt, der für Efraim besteht, ist kein konkretes Einzelereignis, das stattgefunden hat oder nicht, sondern ein genereller Sachverhalt. Er ist zudem eher vage formuliert, sodass es schwierig ist herauszufinden, was genau Efraim eigentlich meint. (Mit dieser Vagheit müssen wir aber leben, weil Efraim nicht allzu tief in die Bände füllende philosophische Problematik einsteigt.) Dass es keine Gesetze gebe, ist allerdings eine Behauptung, die sich offensichtlich nicht auf Naturgesetze bezieht. Gemeint sind vermutlich metaphysische Gesetze, die den Lauf der Welt in irgendeiner Weise vorherbestimmen.

Dass der Mensch der Zufälligkeit der Welt nicht entkommen kann, sondern ihr ausgeliefert ist, ist eine Überzeugung, die Efraim angesichts eines Vorfalls bekräftigt, mit dem er konfrontiert wird, als er - rein zufällig - in der Nachrichtenagentur die Information über einen Doppelselbstmord zweier Schülerinnen durch Gas erhält und sich daraufhin weniger aus eigenem Interesse an den Tatort begibt als aus dem Umstand, dass er sich genötigt fühlt, der Mitteilung durch die Sekretärin Folge zu leisten. Efraim verhält sich also auch so, wie er denkt, d. h. er gibt äußeren Anstößen nach, liefert sich ihnen aus, hat ihnen nichts entgegenzusetzen. Nach alldem, was er tut und sagt, ist er kein willensstarker Mensch.

Am Tatort angekommen, trifft er auf den ermittelnden Kriminalrat von Cantz, Gelegenheit für Efraim, seine Ansichten über die Motivation der Mädchen bzw.

\footnotetext{
${ }^{3}$ Als grobe Orientierung für das hier zugrunde gelegte Konzept mimetischer Unzuverlässigkeit vgl. Kindt (2008, S. 51). Ich fasse es aber insofern enger, als ich Unwahrheit für eine notwendige Bedingung halte und Unvollständigkeit nicht berücksichtige.

${ }^{4}$ Zit. nach der Erstausgabe Andersch 1967 unter der Sigle E. Vgl. auch Andersch, GW, 2.
} 
die Gründe der Tat darzulegen. Die Kinder seien zufällig gestorben, meint er. Ereignisse, die außerhalb der Gewalt der Mädchen lagen, hätten ihre Entscheidung beeinflusst. Hätten andere Lichtverhältnisse in der Zeit vor ihrer Entscheidung geherrscht, wäre es vielleicht nicht zu der Tat gekommen. Kleinste Änderungen von Umweltbedingungen hätten in den Gehirnen die Bereitschaft ausgelöst, den Gashahn aufzudrehen. „Alles ist Zufall“, folgert Efraim (E, 36). Zugleich spekuliert er über die Motivation der Mädchen. Efraim vermutet, dass Langeweile das Motiv gewesen sein könnte, wobei er offenkundig seine eigene Empfindung angesichts der tristen Örtlichkeit auf die Einstellung der Mädchen vor ihrer Tat überträgt. Efraim redet, ohne nachzudenken, und auch das, was er weiter sagt, sind leere Verallgemeinerungen, etwa über das angebliche Selbstmordverhalten der Italiener. Der Kriminalrat korrigiert ihn mit seinem Hinweis auf „spezielle Gründe", die nicht polizeilich relevant sind, aber bei der Tat der Mädchen eine Rolle gespielt haben müssten. Außerdem spricht sich von Cantz gegen die Existenz von Nationaleigenschaften aus, womit er Efraims leeres Gerede entlarvt, was man an dieser Stelle jedoch noch nicht sicher feststellen kann. Der Kriminalrat spielt im weiteren Verlauf von Efraims Geschichte zwar keine Rolle mehr. Allerdings kommt Efraim einmal noch auf ihn zu sprechen, wenn er ihn als einen der ,sympathische[n]“ Deutschen bezeichnet, deren er sich nach seinem Berliner Aufenthalt erinnert (E, 344). Daher spricht einiges dafür, dass von Cantz ebenso als Korrektiv von Efraims Position dient wie Frau Heiß und Werner Hornbostel.

Diese beiden Figuren zeichnen sich dadurch aus, dass sie Efraims Verlautbarungen, die im Zusammenhang mit der Judenvernichtung stehen, widersprechen. Frau Heiß wohnt mit ihrer Familie in dem Haus, das Efraims Eltern gehört hat und das er nun das erste Mal nach seiner Emigration als Jugendlicher aufsucht. Offenbar um Frau Heiß von Schuldgefühlen freizusprechen, sagt Efraim etwas, das man nicht anders als eine mehr als platte, ja provokante Relativierung des Holocaust auffassen muss. Enteignung habe es immer schon gegeben, meint Efraim (E, 46), und marginalisiert damit die massenhafte Tötung von Juden, wofür die Enteignungen nur das Vorspiel waren. Doch Frau Heiß geht darauf nicht ein, sondern besteht auf der Einzigartigkeit des Verbrechens. Ähnlich verläuft der Dialog mit Hornbostel, einem Avantgarde-Musiker, den Efraim über Anna Krystek kennenlernt, die junge Schauspielerin, mit der er viel Zeit in Berlin verbringt. An zentraler Stelle des Romans leugnet Efraim jegliche „Erklärung für Auschwitz“ (E, 229) und besteht auf der Zufälligkeit der Judenvernichtung. Hornbostel, der Sohn eines Nazis, widerspricht, indem er darauf hinweist, dass die Judenvernichtung und jeder einzelne Mord gewollt worden seien. Es folgen, genau in der Mitte des Romans, einmontierte Zitate aus den Frankfurter Auschwitz-Prozessen, die die pure Mordlust der Täter bezeugen (vgl. Schlant 2001, S. 216 f.). Damit vertritt Hornbostel genauso wie Frau Heiß in zwei zentralen Fragen die Gegenposition zu Efraim.

Allein das ist natürlich heikel, denn es scheint historisch unangemessen, dass ausgerechnet Deutsche die richtige Position vertreten und der Jude Efraim die falsche Position. Matthias Uecker (2000, S. 502) zufolge wird damit der jüdische 
„Erzähler zum Demonstrationsobjekt verdinglicht“, und nach Hans-Joachim Hahn (2011, S. 374) „,beschädigt das das Eingedenken“.

Möglicherweise hat Andersch geahnt, dass die Darstellung von Deutschen, die ein angemessenes (d. h. nicht relativierendes bzw. nicht beschönigendes) Verhältnis zu den Greueltaten der Nazis haben, damals nicht gerade repräsentativ für die Bevölkerung war. Im Text findet sich ein Hinweis, der sich als Absicherung gegen die Kritik verstehen lässt, dass gerade deutsche Figuren als Garanten der richtigen Normen fungieren: Efraim wundert sich selbst über die guten einsichtigen Deutschen, die er trifft: „Vielleicht habe ich besonderes Glück gehabt, vielleicht bilden solche Leute nur eine winzige Minderheit" (E, 345). Efraim stellt also die allgemeine Geltung seiner positiven Einschätzung selbst infrage, und der Roman konterkariert damit den potenziellen Eindruck, dass die dargestellten deutschen Figuren repräsentativ für alle Deutschen seien.

Ehe ich zu der Entscheidung komme, welche der vertretenen Ansichten im Roman wahr bzw. richtig ist, fasse ich die beiden Widersprüche, um die es geht, zusammen: Auf der einen Seite steht Efraim, der glaubt, dass der menschliche Wille bei seinen Handlungen keine effektive Rolle spiele und dass historische Ereignisse keinem sinnvollen Plan folgten. Der letzte Punkt kann für Verwirrung sorgen, weil dazu zwei unterschiedliche Überzeugungen gehören, von denen nur eine falsch ist. Efraim scheint zu meinen (und in seinen ersten Äußerungen spricht er es auch aus), dass die Judenvernichtung ein im Prinzip austauschbares Ereignis sei. Dem widerspricht Frau Heiß zu Recht, die demnach die Gegenposition zu Efraim einnimmt. Nach dem Gespräch mit ihr besteht Efraim, nun allein mit sich, allerdings darauf, dass sich dergleichen wiederholen könnte. Das wiederum wird im Roman nicht widerlegt. ${ }^{5}$

Efraim ist ein autodiegetischer Erzähler und als solcher privilegiert. Daher wird man ihm zunächst Glauben schenken wollen. Außerdem hat er die Eigenschaft, jüdisch zu sein, und als Jude ist er mittelbar betroffen von den Gewalttaten der Nazis, denen seine Eltern zum Opfer gefallen sind. Auch das erhöht seine Glaubwürdigkeit (vgl. Feuchert 2016). Zudem trägt er einige Züge, die er mit seinem Autor teilt: etwa die Auffassung über den Stil, die Vorliebe für Beckett oder auch die starke Kurzsichtigkeit. Es ist also höchst kontraintuitiv anzunehmen, dass Efraim unzuverlässig ist, wenn er nicht nur über die Autorität des Juden, sondern auch noch in Teilen über die des Autors verfügt.

\footnotetext{
${ }^{5}$ Damit hat sich die Beurteilung Efraims verschoben. Die (prospektive) Befürchtung, dass sich eine ähnliche Katastrophe wie die Judenvernichtung im Prinzip wiederholen könne, relativiert die Shoa nicht wie die retrospektiven Einlassungen Efraims zuvor. In diesem Zusammenhang erweist sich der zeithistorische Hintergrund, vor dem Efraims Berlinaufenthalt steht, nicht als zufällig gewählt, sondern als bedeutsam: Für die Zeitgenossen stand die Welt Ende Oktober 1962 vor dem atomaren Abgrund. Der Vorwurf, nichts aus der Gefahr der Massenvernichtungen des Zweiten Weltkriegs gelernt zu haben, war angesichts der Konfrontation auf dem Höhepunkt des Kalten Krieges eine reale Einstellung. Zur Funktion der Kubakrise im Roman vgl. Aumüller (2020).
} 
Warum aber sollte man es dem Roman nicht abkaufen, dass das, was Efraim äußert, wahr und richtig ist, sondern das, was seine Gesprächspartner sagen? Hier ist die Antwort: Nicht nur Frau Heiß und Werner Hornbostel widersprechen Efraim, später tut er das auch selbst, wenn er den Tod seiner Eltern mit dem Tod von Anna Krysteks Mutter vergleicht und zu dem Schluss kommt, dass es einen Unterschied bedeute, durch Zyklon B zu sterben anstatt durch einen Bombenabwurf (E, 344). Auch sein spontaner Faustschlag, der sich gegen die unbedarfte Äußerung der Wendung „bis zur Vergasung“ wendet (E, 203), zeugt davon, dass die Judenvernichtung eine Bedeutung für ihn hat, die er lange leugnet. Außerdem ist die Figur des Efraim so angelegt, dass sie eine Entwicklung durchmacht, an deren Ende die Erkenntnis steht, der zufolge man die Wahl hat, sich für oder gegen etwas zu unterscheiden: in diesem Fall den Beruf des Journalisten aufzugeben und ein Buch über sich selbst zu schreiben. Diese Einstellung, zu der Efraim findet, ist zugleich eine von Anderschs wichtigsten Überzeugungen, sodass weit mehr dafür spricht, dass Efraims Zufallstheorie im Roman nicht als richtige Theorie dargestellt wird, sondern als falsche Theorie, die im Verlauf des Buches widerlegt wird.

\section{Die Frage nach der Tradition}

Efraim nimmt eine Sonderstellung in Anderschs Roman-Euvre ein. Narratologisch gesehen, fällt das Werk dadurch heraus, dass es homodiegetisch angelegt ist. Aber auch werkgeschichtlich ist in der Andersch-Forschung von einer Veränderung die Rede: „Anderschs Entdeckung Englands und des englischen Romans in den 60er Jahren markiert einen Wendepunkt sowohl in seinen literarischen Vorlieben als auch in seiner Einstellung zur Form des Romans überhaupt" (Williams 1994, S. 122).

Betrachtet man nur die beiden Romane, die Andersch vor Efraim verfasst hat (Sansibar von 1957 und Die Rote von 1960), so fällt unmittelbar auf, dass die vorherrschende Konzeption das multiperspektivische Erzählen ist, wobei die jeweiligen Ich-Perspektiven als mentale Erzählungen angelegt sind. Efraims Ich-Perspektive ist demgegenüber als bereits erschriebene - und damit in ihrer Unmittelbarkeit gebrochene, weil reflektierte - Perspektive dargeboten. Seine Erzählrede ist als schriftlicher Text konzipiert, der mehrere Überarbeitungsschritte durchlaufen hat. Ein Clou dabei ist, dass der Text zunächst nicht als geschriebener erkennbar ist, sondern als mentale Rede - in der erzählten Welt - fingiert wird. (Auch in dieser Hinsicht ist Efraim ein, wenigstens temporär, unzuverlässiger Erzähler, aber darum geht es jetzt nicht.) Die mentalen Reden der einzelnen Figuren etwa in Sansibar sind - auf der Ebene der Fiktion - tatsächlich innere Monologe; Efraims mentale Rede erweist sich dagegen als - auf der Ebene der Fiktion - fingierter innerer Monolog, der in der Wirklichkeit der Fiktion Ergebnis der Entscheidung Efraims ist, auf konventionelle literarische Mittel beim Erzählen seiner Geschichte zu verzichten.

Wie kam Andersch zu dieser Technik? Ein wichtiger Einfluss könnte Henry James sein, mit dessen Notebooks Andersch sich in der Entstehungszeit von 
Efraim beschäftigt hat (vgl. Andersch und Frisch 2014, S. 61). Allerdings lagen zu dieser Zeit laut Rhys Williams (1994, S. 127) bereits fünf von sieben Kapiteln vor. Daher hält er es für unpassend, von einer „Inspirationslektüre“ für Efraim zu sprechen, und widerspricht damit Stephan Reinhardt (1990, S. 390). Was die Erzählkonzeption angeht, spricht in der Tat einiges dagegen, stand James dem homodiegetischen Erzählen doch eher skeptisch gegenüber. Daher sieht Williams (1994, S. 128) die Bedeutung James’ für Andersch in einer „Bestätigung für die Einsichten über die Aufgabe des Schriftstellers“, die Andersch in James' Notebooks fand. Im Roman selbst spielt James denn auch nicht in poetologischer oder narratologischer Hinsicht eine Rolle, sondern mit Blick auf sein Reise-Buch English Hours von 1905 (vgl. E, 320).

Es gibt einige weitere Autoren, die Efraim erwähnt. Unter ihnen ist Samuel Beckett der prominenteste, und er spielt für ihn eine wichtige Rolle. Immer wieder hat auch Andersch selbst auf seine Bewunderung von Samuel Beckett hingewiesen. Genauso lässt sich George Efraim von Becketts Werk ansprechen: „[S]olche Litaneien der Gleichgültigkeit aus einer Welt des Chaos und des Zufalls sagen mir außerordentlich zu“ (E, 317). Beckett fasst in Worte, was Efraim fühlt und denkt. Hingegen galten Beckett und die an ihn anknüpfende Tradition des Nouveau Roman Andersch nicht als literarisches Vorbild. In einem Brief an Max Frisch vom 25. Februar 1964, als er in Berlin bereits an seinem Roman schrieb, heißt es: „Ich glaube, es gibt wohl keinen grösseren Beckett-Verehrer als mich, aber ich werde niemals einen Versuch machen, mich der ganzen Richtung formal zu nähern“ (Andersch und Frisch 2014, S. 53). Gegenüber dem Philosophen Wilhelm Schapp äußert er sich in einem Brief vom 25. Februar 1963 voller Ablehnung über RobbeGrillet, der meine, „Handlung und Held, Thema und Sujet, seien gänzlich überflüssig, die Zeit des eigentlichen Erzählens sei vorbei [...]. Die ganze Theorie ist meiner Meinung nach Schwachsinn“ (zit. nach Williams 1994, S. 127 f.).

Beckett dient zur Charakterisierung von Efraims Geisteshaltung, die nicht diejenige Anderschs ist. Diese Haltung lässt sich durch ihre Tendenz zum Nihilismus aus Verzweiflung charakterisieren. Beckett ist aber weder für Efraim noch für Andersch ein literarästhetisches Vorbild. Dieser Unterschied entgeht Max Frisch, der wenige Jahre später das fertige Manuskript liest und Andersch seine ausführlichen Lektüreeindrücke schriftlich mitteilt: „Was Efraim da über Beckett schreibt, ist natürlich ausgezeichnet, aber ich bin nicht sicher, ob Efraim da gefragt worden ist über sein Verhältnis zu Beckett; Efraim kommt mir da etwas beschenkt vor" (Andersch und Frisch 2014, S. 73).

Frischs Fokus liegt auf der Differenz zwischen Autor und Protagonist. Er erkennt und weiß wohl auch, dass Efraim nicht als alter ego von Andersch konzipiert ist. ${ }^{6}$ Daher weist er Andersch auf Stellen hin, die eine - nicht zu dieser

\footnotetext{
${ }^{6}$ „Da es sich, wie ich meine, um einen illusionistischen Roman handelt, nicht um einen SpielRoman, ist dem Ich wohl alles erlaubt, was zu ihm gehört, aber kein Plagiat beim realen Autor“ (Andersch und Frisch 2014, S. 78).
} 
Konzeption passende - Identifizierung von Efraims Ansichten mit denen des Autors nahelegen. ${ }^{7}$ So ist auch der Punkt zu verstehen, dass Efraims Vorliebe für Beckett nicht recht zu seinem Charakter zu passen scheint. Frisch sieht in der Beckett-Vorliebe nur die ästhetische Komponente. Es kommt Andersch aber auf die Weltanschauung bzw. das Lebensgefühl an, das sich in Becketts Werken ausdrückt und das genau das ist, von dem sich Efraim identifikatorisch angesprochen fühlt. ${ }^{8}$

Beckett ist also nicht für die poetologische Konzeption von Efraim verantwortlich zu machen. Meiner Ansicht nach sind es Frischs erste zwei Romane, an deren Konzeption sich Andersch orientiert hat. ${ }^{9}$ Einen Hinweis darauf gibt Andersch selbst im Gespräch mit Horst Bienek. Darin preist er Max Frischs von ihm sogenanntes zweifelndes Erzählen: „Max Frisch [...] ist ein gutes Beispiel dafür, wie man heute erzählen kann. Seine Qualität liegt nicht darin, daß er die Tagebuch- und die Ich-Form benutzt, sondern darin, daß er zweifelnd erzählt. Er versteht es unnachahmlich, seine Leser in den Prozeß seiner Erfindung einzubeziehen“ (Bienek und Andersch 1962, S. 120). Nichts anderes lässt Andersch seinen Efraim tun, wenn er den Entstehungsprozess seines Romans durch Efraim selbst dokumentiert. Efraim ist ein zweifelnder Erzähler par excellence und lässt die Leser an der Genese seines Romans teilhaben, indem er die Entstehung des Romans zu einem seiner zentralen Themen macht.

Zugleich ist es gerade die Kombination von Zweifeln und Erzählen, die Andersch offensichtlich besonders attraktiv fand. Während der Zweifel bei Beckett die dominante Größe ist, aber eben auch auf das Erzählen selbst übergreift und es nivelliert, ist es das Festhalten am Erzählen, worauf es Andersch ankommt und worin Frisch ihm zum Vorbild wird. Mit der Kombination von Zweifeln und Erzählen, die er bei Frisch findet, kann er das ästhetische Ziel der Moderne weiter verfolgen, keine wohlfeilen Gewissheiten zu vermitteln, und zugleich an seinem eher unzeitgemäßen Interesse an Psychologie festhalten bzw. daran, eine Geschichte zu erzählen, die von den Beziehungen der Menschen zueinander handelt. Noch einmal Andersch über Frisch: „Er fordert sie [die Leser] auf, alles zu bezweifeln, was er sagt. Aber niemals wäre er bereit, auf das Erzählen zu verzichten [...]“ (Bienek und Andersch 1962, S. 121).

\footnotetext{
${ }^{7}$ Z. B. auch: „Efraims Stil-Dekrete, ich gestehe, dass es mir schwerfällt, darin nicht die StilDekrete von Alfred Andersch zu sehen“ (Andersch und Frisch 2014, S. 66).

${ }^{8}$ Gleichzeitig muss man wohl sagen, dass Frisch hellsichtig auf etwas hingewiesen hat, was man dem Roman dann in der Tat als Mangel ausgelegt hat: Es steckt in Efraim zu viel Andersch, als dass man ihn als eigenständige Figur sehen könnte. - Zu Efraims Vorliebe für Beckett passt im Übrigen nicht, dass er sich selbst an anderer Stelle als „,konservativ“ bezeichnet, „,was die schöne Literatur betrifft" (E, 316). Efraim mag Literatur über die Beziehungen von Menschen untereinander und bevorzugt einen klaren Stil, weil diese Beziehungen ja ,schon differenziert und geheimnisvoll genug" sind.

${ }^{9}$ Mein Name sei Gantenbein kannte Andersch noch nicht, als er Efraim begann (vgl. Andersch und Frisch 2014, S. 52). Er holte die Lektüre aber nach. Im Sommer 1964 las er den Text und äußerte sich darüber mit großer Anerkennung (vgl. Andersch und Frisch 2014, S. 58-60).
} 
Es ist nicht ausgeschlossen, dass neben Frisch weitere Autoren eine Rolle gespielt haben können. Bekannt ist Anderschs Bewunderung italienischer Autoren des Neorealismus. Besonders schätzte er u. a. Elio Vittorini, von dessen Werken er Gespräch in Sizilien (1941, dt. 1948) auch in dem Gespräch mit Bienek hervorhebt. Andersch hält „,den italienischen Neo-Realismus, wie er 1943 zum erstenmal in Vittorinis Roman ,Conversazione in Sicilia“ und Viscontis Film ,Ossessione“ auftrat, für das größte künstlerische Ereignis seit dem zweiten Weltkrieg [...], übertroffen seither höchstens durch das Werk von Samuel Beckett" (Bienek und Andersch 1962, S. 121). Auch wenn der Neorealismus nicht im Verdacht steht, eine besondere Tendenz zum unzuverlässigen Erzählen aufzuweisen, könnte dieses Werk eine gewisse Affinität dazu erkennen lassen, da die homodiegetische Rede passagenweise als Traumrede interpretierbar ist und somit der Realitätsstatus des Erzählten infrage steht. ${ }^{10}$ Allerdings gleicht weder der Charakter der Unzuverlässigkeit selbst noch die funktionale Einbindung in den Roman der narrativen Anlage von Efraim auch nur im Entferntesten. Nicht überraschend hebt Andersch daher in einem Aufsatz über Vittorini weniger diese Qualität des Erzählers von Gespräch in Sizilien hervor als ,eine poésie pure der optischen Härte“, deren besonderes Verdienst er in der Vereinigung zweier ansonsten getrennter Bereiche erblickt: „Abstraktion und Gegenstand, ja Prosa und Lyrik selbst, erscheinen in seinem Werk als Identitäten“ (Andersch 1979 [1959], S. 36; GW 9, 282).

Während Andersch seinen italienischen Kollegen für dessen Kombination zweier künstlerischer bzw. literarischer Repräsentationsweisen lobt, richtet sich das Lob, das er seinem Schweizer Kollegen zollt, auf Eigenschaften von dessen Romankunst, die stärker mit der Konzeption unzuverlässigen Erzählens assoziiert ist als diejenigen, die er bei Vittorini hervorhebt. Daher ist es wahrscheinlicher, dass Frisch mit Blick auf das unzuverlässige Erzählen in Efraim die entscheidende Einflussgröße (von den hier in Betracht gezogenen) ist.

\section{Kognitive und emotionale Funktionen der narrativen Unzuverlässigkeit}

Eingangs habe ich zwei Varianten der Frage danach erwähnt, warum sich Andersch für einen unzuverlässigen Erzähler entschieden hat, von dem man jetzt sagen kann, dass diese Wahl sich wahrscheinlich dem Einfluss Max Frischs verdankt. Die erste Variante fragt danach, was Andersch dazu bewogen haben könnte,

\footnotetext{
${ }^{10}$ Genauer gesagt, erweisen sich die phantastisch anmutenden Erlebnisse, die im 41. bis zum 43. Kapitel erzählt werden, als Resultat eines Rauschs. Da Silvestro, der Ich-Erzähler, vorher mit anderen getrunken hat und sich vor Beginn der Passage und danach auf dem Treppenabsatz des Hauses seiner Mutter befindet, lässt sich aber erkennen, dass diese Passage einen anderen Status besitzt. Vgl. Vittorini (1961, S. 176 ff.). - Zur Bedeutung des Neorealismus für Andersch vgl. Perrone Capano (2012) und Kammandel (2012).
} 
sich dieser Konzeption zu bedienen, und auch darauf kann man nun eine Antwort geben: Es ist das, was Andersch als Kombination von Zweifeln und Erzählen ausgegeben hat. Der unzuverlässige Erzähler dient Andersch dazu, Überzeugungen zu äußern, die von den Lesern in Zweifel gezogen werden sollen. Zugleich wird die erzählte Welt nicht gänzlich aufgehoben oder infrage gestellt. Efraims Fehlbarkeit kann erst auf dem Hintergrund einer stabilen Welt überhaupt sinnvoll als solche wahrgenommen werden. Und der wichtigste Teil der erzählten Welt ist für Andersch das individuelle Bewusstsein eines jeden Menschen. Anderschs Credo besteht darin, dass er der jeweils individuellen menschlichen Psyche einmalige Realität zuerkennt und dass es der Roman ist, der diese Realität festhält: ,,...] die sogenannte Krise des Romans besteht in nichts anderem als in der Frage, ob dem Menschen noch psychische Realität zugestanden wird oder nicht", formuliert Andersch 1969 in seiner Laudatio auf Giorgio Bassani (GW, 9, 456). Der Roman habe „seit Jahrhunderten dieses innere Leben sichtbar gemacht“ (GW, 9, 456). Das Mittel und zugleich die Aufgabe des Romans ist für Andersch die Erinnerung: „Die Aufgabe von Literatur ist es - und ich spreche jetzt von Prosaliteratur -, in erzählerischen Prozessen eine Erinnerung zu formen“ (GW, 9, 455). Insofern die Erinnerung für Andersch eine Aufgabe ist, setzt er sie gegen die von marxistischer Seite vorgebrachte Aufgabe von Literatur, politische Prozesse zu beeinflussen; insofern die Erinnerung ein oder das Mittel der Literatur ist, schöpft sie aus dem persönlichen Reservoir von Erinnerungen des Autors, aber auch aus seiner Einbildungskraft. Efraim lässt sich vor diesem Hintergrund als Versuch verstehen, denjenigen, denen ihre Stimme durch die Judenvernichtung genommen wurde, eine Stimme in der deutschen Nachkriegsliteratur zu geben. Das wurde ihm von vielen Seiten zwar nicht abgenommen (vgl. Reich-Ranicki 1967; Klüger 1998). Und dass diese Stimme überdies unzuverlässig ist, kann Leser auch heute noch verstören. Aber das war das Mittel, das es Andersch ermöglichte, keine vorgefertigten Weisheiten formulieren zu müssen. So umstritten und teilweise auch geschmacklos Andersch seine jüdische Figuren aus heutiger Sicht dargestellt haben mag, man muss ihm doch zu Gute halten, dass er sich als einer der wenigen damals mit dieser Frage überhaupt auseinandergesetzt hat. ${ }^{11}$

Abschließend komme ich zu der Frage, welche Funktionen das Verfahren im Roman im Einzelnen erfüllt. Damit ist eine Untersuchung intendiert, die über das, was der Autor an interpretatorisch verwertbaren Hinweisen gegeben hat, insofern hinausgeht, als sie die einzelnen Eigenschaften und Funktionen benennt, die in der Romanstruktur mit Blick auf Efraims Unzuverlässigkeit und das Zufallsmotiv salient sind. Efraims Zufallstheorie

\footnotetext{
${ }^{11}$ Das gilt selbst dann, wenn seine Motivation eher von egoistischen als von altruistischen Überlegungen geleitet war (etwa weil er sein schlechtes Gewissen entlasten musste, das er vermutlich hatte, da er seine erste Frau samt Familie durch seine Scheidung in Lebensgefahr gebracht hatte, die seine Schwiegermutter auch tatsächlich nicht überlebte). Er hätte ja wie die meisten das Thema einfach verschweigen können. Aber das eben wollte er nicht. Vgl. z. B. HeidelbergerLeonard (1994).
} 
1. ist Ausgangspunkt eines Erkenntnisprozesses, der durch die Auflösung der Unzuverlässigkeit an sein Ende gelangt;

2. dient als Bemäntelung und Überspielung der persönlichen Verzweiflung über die Shoah;

3. hat in der Sinnhaftigkeit der Welt ihren Gegenbegriff, und die Opposition dient dazu, dass sich jedes Individuum dazu in Beziehung setzt; Efraims Zufallstheorie dient nicht dazu, eine besondere Theorie (oder ihr Gegenteil) über die Judenvernichtung zu propagieren, sondern durch die Unzuverlässigkeit ihrer Darstellung ist es ihre Aufgabe, den Umgang mit verschiedenen Erklärungsansätzen der Judenvernichtung überhaupt nachzudenken.

\subsection{Die Zufallstheorie als Ausgangspunkt eines Erkenntnisprozesses}

Gemäß der ersten Funktion dienen Efraims - als unzuverlässig zu erachtende Äußerungen über den Zufall dazu, den Ausgangspunkt einer Entwicklung, die Efraim durchläuft, zu markieren. Als die Haupthandlung einsetzt - der Journalist Efraim befindet sich zu Recherchezwecken in Berlin, wo er offiziell über die deutschen Reaktionen auf die Kuba-Krise berichten und inoffiziell (angeblich, muss man einfügen, weil sein Chef sich ihm, dem jüdischen Emigranten gegenüber, vermutlich auf diese Weise zu seinem Versagen bekennen will) das Schicksal der unehelichen Tochter seines Chefs aufklären soll -, befindet sich der 42jährige Efraim in einer persönlichen Krise. Er weiß bereits, dass er seinen Beruf aufgeben möchte, aber er weiß eigentlich nicht, was er stattdessen tun soll. Zunächst aus Gründen der Selbstvergewisserung beginnt er, sich Notizen über seine persönlichen Erlebnisse zu machen, und scheitert mit dem Versuch, seine Erlebnisse in einer literarisierten Darstellungsweise wiederzugeben (heterodiegetisch und im Präteritum). Man erfährt nach und nach, dass er verheiratet ist, aber schon lange von seiner Frau getrennt lebt. Als Auslandskorrespondent hält er sich vorwiegend in Rom auf, während seine Frau Meg in London wohnt. Vergleicht man die Efraim hauptsächlich bewegenden Erfahrungen seines Lebens - die Trennung von Meg und die Judenvernichtung, der auch seine Eltern zum Opfer gefallen sind - vom Ende des Romans her mit dem, wie Efraim anfangs darüber denkt, so fällt auf, dass er zunächst beide für ihn eigentlich höchst leidvolle Erfahrungen herunterspielt. Das kommt nicht zuletzt in den (oben bereits erwähnten) Gesprächen zum Ausdruck, die er in Berlin führt und die zeitlich vor seinen Reflexionen liegen, in denen er über seine Empfindungen Rechenschaft abzulegen versucht.

Eine weitere Besonderheit an Efraims Text ist der mehrstufige Entstehungsprozess, dessen Darstellung Efraim ins Manuskript übernimmt. Auf diese Weise werden seine Erlebnisse, die der Haupthandlung - wenige Tage im Oktober und November 1962 - angehören, mit den späteren Erlebnissen zur Zeit der Niederschrift des Romans, die sich bis 1965 hinzieht, kombiniert. Während des Abfassens schöpft Efraim aus Notizen, die er jeweils kurz nach den Erlebnissen 
im Jahr 1962 angefertigt hat. Das hat zur Folge, dass nicht immer feststellbar ist, wann er welche Einsicht gewonnen hat, ob gleich unter dem Eindruck seines Berlinaufenthaltes oder erst später während des Schreibens und Überdenkens.

Dennoch kann man sagen, dass seinen Verlautbarungen eine Entwicklung ablesbar ist. Mit Bezug auf seine Beziehung zu Meg besteht die Entwicklung in dem Eingeständnis, dass er ihr noch immer zugetan ist und sich von ihr die Exklusivität ihrer Gefühle für sich wünscht, die sie ihm jedoch nicht geben kann. Damit einher geht die gedankliche und schriftliche Verarbeitung ihres ihn demütigenden Verhältnisses mit Keir Horne, seinem Chef. Hier besteht die Entwicklung darin, dass er etwas, das ihn offenbar schon lange bewegt und womit er noch längst nicht abgeschlossen hat, reflektiert und niederschreibt.

Das ist mit Bezug auf das Zufallsthema nicht so. Er erwähnt es schon sehr früh, als er sich selbst Keir gegenüberstellt. In diesem Fall mündet der Entwicklungsprozess nicht in ein Eingeständnis von etwas, das Efraim schon weiß und nun nur schreibend ausspricht, sondern er besteht in einem Zugewinn von Erkenntnis oder Einsicht von etwas, das ihm vorher nicht klar war. Die Überzeugung, dass alles sinn- und zwecklos sei, hat Efraim 1944 gewonnen, als er von Keir über die Massentötungen der Juden informiert worden war (E, $89 \mathrm{f}$.). Dadurch steht seine sog. Zufallstheorie in einem ursächlichen Zusammenhang mit der Shoah. Die Ursache dafür, dass sich in Efraim die Überzeugung herausbildet, alles beruhe auf Zufall, ist die Erfahrung, dass die Nazis massenhaft Juden vergasen.

Durch die Erlebnisse in Berlin und ihre schriftliche Verarbeitung distanziert sich Efraim zunehmend von der mit seiner Zufallstheorie einhergehenden Relativierung des Holocaust. Der Faustschlag, zu dem er auf einer Party ausholt, als er die unbekümmert geäußerte Redensart „bis zur Vergasung“ zur Beschreibung einer wilden Nacht vernimmt (E, 203), zeugt davon, dass Efraim keineswegs so empfindungsarm bzw. zynisch ist, wie er zunächst tut. Durch den Faustschlag weckt er das Interesse der jungen Schauspielerin Anna Krystek, deren Bekanntschaft er selbst wiederum als Auslöser für sein Buch ansieht. Einige weitere Erlebnisse führen dazu, dass sich Efraim von der Zufallstheorie nach und nach abwendet: die Widerrede von Frau Heiß und Werner Hornbostel, die Begegnung mit Schwester Ludmilla und dem Grabstein der Luise Zoufal, die Erleuchtung auf dem Russell Square, die dazu führt, dass Efraim das macht, wozu er sich berufen fühlt (nämlich ein Buch zu schreiben), die Anerkennung des Unterschieds, der zwischen den Todesarten besteht: wie Annas Mutter im Bombenhagel zu sterben und wie seine Eltern und Millionen anderer vergast zu werden. Schließlich endet das Buch mit der von Efraim selbst so bezeichneten „Marotte“, also der Zufallstheorie, die er offenbar - nun am Ende seines Buches angelangt als widerlegt betrachtet: ,[...] wenn alles auf Zufall beruhte, [...] dann wäre es nicht nötig gewesen, mich so zu demaskieren, wie ich mich in der Tat demaskiert habe“ (E, 468 f.). Die Zufallstheorie erweist sich spätestens hier als falsche Theorie, an die zu glauben Efraim nun aufgegeben hat.

In welchem Verhältnis steht Efraims narrative Unzuverlässigkeit zu Anderschs Einstellung? Jean Améry (2003 [1967], S. 152) hat in seiner wohlmeinenden Rezension prophezeit, „daß Anderschs Efraim ein ,Ereignis“ sein wird, wie vor 
Jahr und Tag Frischs Stiller, wie Bellows Herzog." Damit lag er, wie man heute sagen muss, nicht ganz richtig. Amérys allgemeine Einschätzung des Romans deutet zudem auf ein zumindest partielles Missverständnis hin, denn er sieht ,im Efraim ein Werk des ,Desengagements““, das ,,vorgezeichnet ist in Anderschs persönlichem und politischem Schicksal“ (Améry 2003 [1967], S. 154). Nach Améry spricht aus den früheren Werken Anderschs sein Engagement für die Linke; hingegen aus Efraim ein „Privatismus“ der „eine Absage an die Politik“ darstelle (Améry 2003 [1967], S. 156). Das ist insofern ein Missverständnis, als Efraim nicht das Sprachrohr Anderschs ist, Andersch also nicht alle Überzeugungen Efraims teilt, sondern ihm Überzeugungen in den Mund legt, um etwas anderes zu zeigen. Keineswegs spricht Andersch sich grundsätzlich gegen politisches Engagement aus. Stattdessen liegt der Sinn von Efraims Lern- oder Erkenntnisprozess darin, dass er für sich selbst beschließt, einer ihm angemessenen Betätigung nachzugehen. Was daraus sprechen mag, ist die Überzeugung, dass sich die Kunst nicht zur Anwältin einer politischen Überzeugung machen, sondern ihren eigenen Gesetzen und Bedürfnissen folgen sollte. Es ist gerade das desengagierte Verhalten Efraims, das Andersch mithilfe des unzuverlässigen Erzählens darstellt und in dieser Figur kritisiert. Efraims vorgeschobene Gleichgültigkeit ist nichts anderes als Desengagement, und sein Entschluss, ein Buch zu schreiben, das Gegenteil davon. Engagement für Andersch ist mithin nicht immer politisch, sondern das Richtige für sich selbst zu finden.

Schon zum Journalismus ist Efraim mehr durch Zufall als durch eine positive Willensentscheidung gekommen. Wie er selbst bekennt, war es die Möglichkeit, die Front zu verlassen, die ihn dazu bewegte, einem Aufruf von Keir Hornes Propagandaabteilung zu folgen. Das erste, was er auf der neuen Stelle erfuhr, waren die Nachrichten über die Vernichtungslager. Seitdem lebte Efraim mit seiner Zufallstheorie, die ihn vor der Mühe bewahrte, sich selbst für oder gegen etwas entscheiden zu müssen.

Möglicherweise überträgt der Nichtjude Andersch seinen eigenen Erkenntnisgewinn - unter umgekehrten Vorzeichen - auf Efraim, den Juden. Was Andersch zuerst mit seiner Desertion erlebt hat (nämlich wie sinnvoll es ist, sein Schicksal in die eigene Hand zu nehmen), überträgt er auf seine Wahrnehmung des Schicksals der Juden. Mit Efraim weist er die frühere Schicksalsergebenheit zurück. Während Heidelberger-Leonard (1994, S. 55) zufolge die Lehre in einem älteren Text wie Biologie und Tennis lautet, dass keiner „sich etwas vorzuwerfen“ habe und Andersch „das böse Schicksal“ verantwortlich mache, ändert sich seine Einstellung später: „Anderschs damalige Schicksalsergebenheit sollte sich spätestens mit Efraim in ihr Gegenteil verkehren" (Heidelberger-Leonard 1994, S. 55). Als sei es auf Efraim gemünzt, schreibt Andersch in seinem Essay über Jean Améry: „Daß man sich selbst infrage stellen muß, enthebt niemanden der Notwendigkeit, sich schließlich entscheiden zu müssen“" (Andersch 1979 [1971], S. 130; GW 10, 122). Und noch in seinem letzten Interview im Januar 1980 mit Jürg Acklin spricht sich Andersch gegen eine deterministische Weltanschauung aus, ,welche die Freiheit des Willens leugnete“ und damit „die Freiheit des menschlichen Denkens, die Fähigkeit des Menschen, zu wählen“ (zit. n. Reinhardt 1994, S. 34). 


\subsection{Die Zufallstheorie als Bemäntelung der eigenen Verzweiflung}

Eng verbunden damit ist die zweite Funktion, die die Zufallstheorie im Roman hat. Sie ist nicht nur eine Theorie über den Lauf der Welt, sondern hat auch eine konkrete Funktion im Zusammenhang mit Efraims Seelenhaushalt. Mit ihr versucht Efraim, das Leid zu verdrängen, das er angesichts der Shoah empfindet. So sieht es auch Irene Heidelberger-Leonard (1986, S. 150): „Seine Zufallsphilosophie leistet ihm sozusagen erste Hilfe; sie schützt ihn lange davor, sich seiner eigenen Zerrissenheit zu stellen." Es gibt in dem Roman eine wichtige Episode, die dieses Verhältnis ikonisch darstellt, und zwar Efraims Begegnung mit einem Grabstein auf dem böhmischen Friedhof in Neukölln, dessen Inschrift zugleich eines der Motti des Romans abgibt. Begraben liegt dort Luise Zoufal, eine Emigrantin wie er, die als Protestantin in der Zeit der Gegenreformation mit ihren Glaubensgenossen von Preußen aufgenommen wurde.

Ihr Name erinnert Efraim natürlich an das Thema, das ihn ständig umtreibt: die Zufälligkeit und Austauschbarkeit der Ereignisse, selbst der grausamsten. Aber ihr Name ist ebenso zweideutig wie der Sinnspruch, über den er mit seiner Begleiterin Anna Krystek nachdenkt; nur dass es keinen Hinweis darauf gibt, dass er die verborgene Bedeutung des Namens erkennt. Während der Deutsche zufällig das Wort „Zufall“ in „Zoufal“ sieht, geht der Name - wie häufig im Tschechischen - auf ein von einem Verb abgeleitetes Partizip zurück. Es bedeutet, verzweifelt'. Der Name bildet eine erstaunliche Äquivalenz zum emotionalen Zustand Efraims. Wie die durch das Schriftbild geweckte Assoziation mit dem deutschen Wort die eigentliche tschechische Bedeutung verdeckt, so bemäntelt Efraim mit seiner vordergründigen, banalen Zufallstheorie seine eigene Verzweiflung.

\subsection{Extratextuelle Funktionen der Zufallstheorie}

Die dritte Funktion der unzuverlässigen Darstellung der Zufallstheorie besteht darin, dass die über weite Teile des Textes unaufgelöste Opposition zwischen Zufall und Sinn die Leser zur Reflexion über das Verhältnis anhält. Die weitgehende Unabgeschlossenheit der Darstellung ist ein Kennzeichen moderner Literatur im engen epochengeschichtlichen Sinne des Begriffs, in deren Traditionszusammenhang sich der Text durch seine deutlich irritationsästhetische Konzeption einreiht. Diese Funktion lässt sich also schlüssig mit den Ergebnissen des vorangegangenen Abschnitts über die Frage nach der Tradition in Verbindung bringen. Efraims Umgang mit der Zufallstheorie, sein störrisches Beharren darauf in der Konfrontation mit anderen Figuren während des Berlinaufenthalts und seine Reflexionen später während der Niederschrift, ist eine Umsetzung des „Zweifelnden Erzählens“, das geeignet ist, entsprechende Zweifel auch in der Lektüre zu wecken. 
Die Funktion, Irritation auszulösen, ist nicht auf den Umstand von Efraims Unzuverlässigkeit beschränkt, sondern der gesamten Erzählkonzeption ohne Schwierigkeiten abzulesen. Im Einklang damit steht auch eine letzte Überlegung. Im ersten Abschnitt habe ich behauptet, dass ausgerechnet zwei deutsche Figuren, Frau Heiß und Werner Hornbostel, Efraim widersprechen und damit zu Zeugen für die Unzuverlässigkeit des Erzählers werden. Diese Konstellation wurde als ästhetischer Mangel des Romans empfunden (Uecker 2000; Hahn 2011). Die Stoßrichtung der Kritik ist die, dass die daraus resultierende Figurencharakterisierung unangemessen sei, weil ausgerechnet deutsche Figuren sich als weltanschaulich progressiver erweisen als die jüdische Figur. Man könnte aber zur Verteidigung einwenden, dass es Andersch gerade dadurch, dass er seinen jüdischen Protagonisten fehlbar gemacht hat, gelungen ist, eine heterogene Figur zu erfinden, die Stärken und Schwächen hat und - das sollte nicht vergessen werden - einen Lernprozess durchmacht. Es kommt also darauf an: Wenn historische Repräsentativität die leitende Norm ist, die man für die Einschätzung ansetzt, dann ist Efraim als Figur tatsächlich fragwürdig; ist die leitende Norm so etwas wie psychologische Interessantheit, könnte man die Figur des Efraim für gelungen halten. ${ }^{12}$

\section{Literatur}

Améry, Jean: Efraim - oder die kluge Skepsis. In: J. A.: Werke, Bd. 5: Aufsätze zur Literatur und zum Film. Hg. Hans Höller. Stuttgart: Klett-Cotta, 2003, 151-157.

Andersch, Alfred: Efraim. Zürich: Diogenes, 1967. [= E].

Andersch, Alfred: Gesammelte Werke. Bde. 1-10. Hg. Dieter Lamping. Zürich: Diogenes, 2004. $[=\mathrm{GW}, 1-10]$.

Andersch, Alfred: Nachricht über Vittorini [1959]. In: A. A.: Die Blindheit des Kunstwerks. Literarische Essays und Aufsätze. Zürich: Diogenes, 1979, 28-39.

Andersch, Alfred: Giorgio Bassani oder vom Sinn des Erzählens [1969]. In: GW, 9, 443-456.

Andersch, Alfred: Anzeige einer Rückkehr des Geistes als Person [1971]. In: A. A.: Die Blindheit des Kunstwerks. Literarische Essays und Aufsätze. Zürich 1979, 125-141.

Andersch, Alfred/Max Frisch: Briefwechsel. Hg. Jan Bürger. Zürich: Diogenes, 2014.

Aumüller, Matthias: Entstörung durch Erzählen? Die unausgesprochene Traumatisierung des Erzählerprotagonisten durch die Judenvernichtung in Alfred Anderschs „Efraim“. In: Traumaerfahrungen und Störungen des ,Selbst'. Mediale und literarische Konfigurationen lebensweltlicher Krisen. Hg. vonCarsten Gansel. Berlin/Boston: de Gruyter, 2020, 229-242.

\footnotetext{
${ }^{12}$ Dieser Beitrag enthält Teilergebnisse, die ich in dem vom Schweizerischen Nationalfonds SNF großzügig geförderten Projekt „Literaturgeschichte, Interpretationstheorie und Narratologie. Über ihr Zusammenwirken am Beispiel des unzuverlässigen Erzählens im deutschsprachigen Nachkriegsroman" mit Tom Kindt an der Universität Fribourg erarbeitet habe. Die gesamten Ergebnisse werden in einem Kapitel einer Monographie nach Abschluss des Projekts dokumentiert. Dort findet sich nicht nur eine ausführlichere Analyse von Efraims Unzuverlässigkeit, die noch auf weitere Bereiche des Textes ausgreift, sondern auch einige bislang kaum beachtete Textdetails sowie Hinweise auf die hier aus Platzgründen nicht berücksichtigte, quantitativ jedoch nicht unerhebliche Sekundärliteratur zu Efraim.
} 
Bienek, Horst/Alfred Andersch. Interview. In: Horst Bienek: Werkstattgespräche mit Schriftstellern. München: Hanser, 1962, 113-124.

Feuchert, Sascha: Realitätsreferenzen, inadäquate Erzähler und verantwortungsfreie Zonen: $\mathrm{Zu}$ Alfred Anderschs Roman Efraim im Kontext des Diskurses der Holocaust- und Lagerliteratur. In: Alfred Andersch. Engagierte Autorschaft im Literatursystem der Bundesrepublik. Hg. Norman Ächtler. Stuttgart: Metzler, 2016, 163-177.

Fricke, Harald: Norm und Abweichung. Eine Philosophie der Literatur. München: Beck, 1981.

Hahn, Hans-Joachim: Andersch, Klüger, Sebald: Moral und Literaturgeschichte nach dem Holocaust - Moral im Diskurs. In: Alfred Andersch revisited. Werkbiographische Studien im Zeichen der Sebald-Debatte. Hg. Jörg Döring/Markus Joch. Berlin/Boston: de Gruyter, 2011, 357-379.

Heidelberger-Leonard, Irene: Alfred Andersch: Die ästhetische Position als politisches Gewissen. $\mathrm{Zu}$ den Wechselbeziehungen zwischen Kunst und Wirklichkeit in den Romanen. Frankfurt/M.: Lang, 1986.

Heidelberger-Leonard, Irene: Erschriebener Widerstand? Fragen an Alfred Anderschs Werk und Leben. In: Alfred Andersch. Perspektiven zu Leben und Werk. Hg. Irene HeidelbergerLeonard/Volker Wehdeking. Opladen: Westdt. Verl., 1994, 51-61.

Kammandel, Verena: Die produktive Rezeption zeitgenössischer italienischer Erzähler in der westdeutschen Nachkriegsliteratur. Studien zum Werk von Alfred Andersch und Hans Erich Nossack. Heidelberg: Winter, 2012.

Kindt, Tom: Unzuverlässiges Erzählen und literarische Moderne. Eine Untersuchung der Romane von Ernst Weiß. Tübingen: Niemeyer, 2008.

Klüger, Ruth: Zeugensprache: Koeppen und Andersch. In: Deutsche Nachkriegsliteratur und der Holocaust. Hg. Stephan Braese et al. Frankfurt/M.: Campus, 1998, 173-181.

Perrone Capano, Lucia: „Die ausstrahlende Kraft des Neorealismus“. Neorealistische Bilder und Schreibweisen in der deutschen Nachkriegsliteratur. In: Realismus nach den europäischen Avantgarden. Ästhetik, Poetologie und Kognition in Film und Literatur der Nachkriegszeit. Ästhetik, Poetologie und Kognition in Film und Literatur der Nachkriegszeit. Hg. Claudia Öhlschläger et al. Bielefeld: transcript, 2012, 87-107.

Reich-Ranicki, Marcel: Sentimentalität und Gewissensbisse. In: Die Zeit, Nr. 44 vom 3.11.1967.

Reinhardt, Stephan: Alfred Andersch. Zürich: Diogenes, 1990.

Reinhardt, Stephan: Ästhetik als Widerstand - Andersch als Bürger und engagierter Schriftsteller. In: Alfred Andersch. Perspektiven zu Leben und Werk. Hg. Irene Heidelberger-Leonard/Volker Wehdeking. Opladen: Westdt. Verl., 1994, 32-41.

Schlant, Ernestine: Die Sprache des Schweigens. Die deutsche Literatur und der Holocaust. Deutsch von Holger Fliessbach. München: Beck, 2001.

Sebald, W. G.: Der Schriftsteller Alfred Andersch. In: Ders.: Luftkrieg und Literatur. München: Hanser, 1999 [zuerst in Lettre International 20 (1993)], 121-160.

Uecker, Matthias: „Das Verhältnis dieser Leute zu uns hat ja auch wirklich etwas Obszönes angenommen." Juden und Deutsche in Alfred Anderschs Roman Efraim. In: Jews in German Literature since 1945. Hg. Pól O’Dochartaigh. Amsterdam/Atlanta: Rodopi, 2000, 491-505.

Vittorini, Elio: Gespräch in Sizilien. Durchgesehene Übersetzung von Werner Haftmann. Olten/ Freiburg i. Br.: Walter, 1961 [1948].

Williams, Rhys W.: Andersch, Efraim und England. In: Alfred Andersch. Perspektiven zu Leben und Werk. Hg. Irene Heidelberger-Leonard/Volker Wehdeking. Opladen: Westdt. Verl., 1994, $122-130$.

Zymner, Rüdiger: Funktionen der Lyrik. Münster: Mentis, 2013. 
Open Access Dieses Kapitel wird unter der Creative Commons Namensnennung 4.0 International Lizenz (http://creativecommons.org/licenses/by/4.0/deed.de) veröffentlicht, welche die Nutzung, Vervielfältigung, Bearbeitung, Verbreitung und Wiedergabe in jeglichem Medium und Format erlaubt, sofern Sie den/die ursprünglichen Autor(en) und die Quelle ordnungsgemäß nennen, einen Link zur Creative Commons Lizenz beifügen und angeben, ob Änderungen vorgenommen wurden.

Die in diesem Kapitel enthaltenen Bilder und sonstiges Drittmaterial unterliegen ebenfalls der genannten Creative Commons Lizenz, sofern sich aus der Abbildungslegende nichts anderes ergibt. Sofern das betreffende Material nicht unter der genannten Creative Commons Lizenz steht und die betreffende Handlung nicht nach gesetzlichen Vorschriften erlaubt ist, ist für die oben aufgeführten Weiterverwendungen des Materials die Einwilligung des jeweiligen Rechteinhabers einzuholen. 